\title{
Classification of Texture Images using Multi-scale Statistical Estimators of Fractal Parameters
}

\author{
Sébastien DeGuY ${ }^{1} \quad$ Christophe DEBAIN ${ }^{2}$ \\ Albert BENASSI ${ }^{3}$ \\ ${ }^{1}$ Université d'Auvergne, LLAIC1, IUT Département Informatique, BP86 \\ 63172 Aubière Cedex, France. \\ ${ }^{2}$ CEMAGReF Clermont-Ferrand, 24 avenue des Landais, BP 50085 \\ 63170 Aubière Cédex, France. \\ ${ }^{3}$ Université Blaise Pascal, LaMP, Pôle physique, Complexe Scientifique des Cézeaux \\ 63177 Aubière Cédex, France.
}

\begin{abstract}
We present a new method of fractal-based texture analysis, using the multiscale fractional Brownian motion texture model, and a new parameter, intermittency. The intermittency parameter $p$ describes a degree of presence of the textural information: a low value of $p$ implies a very lacunar texture. The multi-scale fractional Brownian motion model allows to construct multiregime textures in the frequency domain. Adding intermittency to this model, we compose the intermittent multi-scale fractional Brownian motion model: the Hurst and intermittency parameters of such processes are functions $H(l)$ and $p(l)$ depending on a scale $l$. The texture is thereby seen as the fusion of structures and details. The structure of the texture is analyzed with the large values of $l$, corresponding to the low frequency content of the texture. The details of the texture are analyzed with the small values of $l$, related to the high frequency content of the texture. The texture is then characterized by all the estimated values of $H(l)$ and $p(l)$, for all the scales $l$ of analysis. The method allows a multi-frequency analysis, permitting the choice of significant scales in a classification task. An application to the classification of corn silage texture images, for which the low frequency content is determining, is proposed.
\end{abstract}

\section{Keywords.}

Texture, fractal, multi-scale and multi-frequency analysis, multi-scale fractional Brownian motion, intermittency, Hurst parameter, statistical estimators, self-similarity, image classification.

\section{Introduction}

In texture analysis using a fractal approach, the fractional Brownian motion ( $\mathrm{fBm}$ ) model [12] is one of the most commonly used: the texture is viewed as the realization of a 
random process. To characterize the texture, the fractal dimension of the underlying fractional Brownian motion's graph has to be estimated. This approach is successful, but suffers from several limitations: from a practical point of view, the fractal dimension estimation methods are very versatile; from a more conceptual point of view, the textures are not always perfectly fractal.

In this paper, we use a generalization of the fractional Brownian motion called multiscale fractional Brownian motion [3] (msfBm) and a new fractal parameter called intermittency [1]. The msfBm model takes into account multi-frequency fractal regimes, and the intermittency parameter gives information on the homogeneity of the texture. Combining these two contributions, the intermittent multi-scale fractional Brownian motion (imsfBm) model of texture can be used.

To test the validity of this approach, we worked with natural corn silage texture images. The aim is to classify eight different silage types. The algorithm firstly "learns" the different types, and then automatically classifies incoming images, through a minimum at the center of gravity of the class.

In the first part of this paper, we introduce our new approach, describing the intermittency parameter, the multi-scale fractional Brownian motion, and a multi-scale estimation of the imsfBm parameters. The benefits of this approach are set out. In the second part, we apply this new method to a real texture classification task: the classification of corn silage texture images. In the last part, we conclude and suggest possible perspectives for improving this promising technique.

\section{The IMSfBM Texture Model}

Limitations of the Fractional Brownian Motion Model. This texture model is dependent on the Hurst parameter, taking values between zero and one and linearly related to the fractal dimension of the graph of the fractional Brownian motion (fBm). A texture, seen as the realization of a $\mathrm{fBm}$, is characterized through the estimation of the value of this parameter, and knowledge of the Hurst parameter value affords almost complete information about the process. Therefore, all the fBm-based studies focus on an estimation of the Hurst parameter and several estimators were set up to this end [5].

Introduced in [2], the estimator of the Hurst parameter of fBm processes we use, is presented in equation 1 . Let $N$ be the number of pixels in the analyzed texture image, $l$ the scale step, and $V_{N, l}^{r}$ the $2 r$-variations of $X$ given by $V_{N, l}^{r}=\sum_{k}\left[X\left(\frac{k+l}{N}\right)-X\left(\frac{k}{N}\right)\right]^{2 r}$, we can write the estimator of the Hurst parameter:

$$
\widetilde{H}=-\frac{1}{2 \log N} \log \frac{V_{N, l}^{1}}{V_{N, l}^{2}},
$$

The slope of the estimation curve $\left(\frac{1}{2} \log \frac{V_{N, l}^{1}}{V_{N, l}^{2}} ; \log \frac{l}{N}\right)$ gives the estimated value of $H$. This slope is computed by a linear regression for few small scales.

The $\mathrm{fBm}$, and its characterization by estimation of its Hurst parameter value, is largely used in texture analysis, e.g., $[8,14,10,11,13,4]$. However, the $\mathrm{fBm}$ is an homogeneous process (the process is statistically the same on the whole space), and its high and low frequency contents are equivalent (the process is the same whatever the scale of observance). Hence, the $\mathrm{fBm}$ is not a very flexible model of texture. 
In addition, for all these studies, the estimation of $H$ encounters an empirical choice of scales: the Hurst parameter is generally obtained by linear regression, computing the slope of the estimation curve according to several scales. Thereby, all authors using this technique are confronted with the same problem: the estimation curve is not linear.

A slight change in the choice of the scales of estimation dramatically modifies the results, even on synthetic images. In [9], the problem is recognized, and the author proposes an algorithm, choosing the scales for which the curve is almost right. However these scales are generally chosen between 1 and a small number ( 5 for example).

This upper bound depends on the texture, so the texture can be partly characterized by this rupture. This justifies enlarging the range of scales used in our analysis, to take into account the whole estimation curve: in general, the values obtained by a linear regression have no physical significance, though the curve shapes do.

Intermittency. It is firstly possible to improve the $\mathrm{fBm}$ model, introducing inhomogeneity through a new parameter called intermittency.

The intermittency parameter $p$, introduced in [1], represents the probability that, at frequency $2^{j}$, the texture has $\delta_{j} \simeq\left[2^{d} p\right]^{j}$ "objects" of spatial size $2^{-j}$. Thereby, a low value of the intermittency parameter creates lacunar zones in the signal, and this information along the scales characterizes the texture under study. The estimator of the intermittency parameter $p$, introduced in [1], takes into account this information:

$$
\log \delta_{n}=-\frac{1}{n} \log \frac{V_{N, l}^{2}}{\left(V_{N, l}^{1}\right)^{2}}
$$

The exponential of the slope of the estimation curve $\left(\log \frac{V_{N, l}^{2}}{\left(V_{N, l}^{1}\right)^{2}} ; \log \frac{l}{N}\right)$ gives the estimated value of $p$.

The Intermittency and Hurst parameters do not give the same textural information, and are independant $[1,7]$. For a value of $p=1$, however, the signal obtained is a fBm with Hurst parameter $H$. Simulations and estimations of intermittent signals are presented in [1].

Intermittent Multi-scale Fractional Brownian Motion. It is secondly possible to construct processes taking into account changes of regimes along the scales. Multi-scale fractional Brownian motion (msfBm) was introduced in [3] for that purpose. This process is a generalization of the $\mathrm{fBm}$ with the Hurst parameter $H$ value varying with frequency, becoming a function $H(l)$, depending on the frequency $\frac{1}{l}$, at scale $l$ (hereafter, we use the terms high frequencies for small scales $l$, and low frequencies for large scales). In bio-mechanic analysis for example, situations like this, where $l$ is a time, are encountered [6].

Allowing intermittency to the msfBm process, we involve the intermittent multi-scale fractional Brownian motion (imsfBm) model. Thereby, any imsfBm-based texture can be seen as the superimposition of a structural organization (at large scales), and textural information (at small scales), each of these informations possibly being inhomogeneous.

The estimators of $H(l)$ and $p(l)$ remain the same. The curves are merely extended to the large scales to collect the two regimes of the process (we then see two slopes [3]). 


\begin{tabular}{|c|c|c|c|c|}
\cline { 2 - 5 } \multicolumn{1}{c|}{} & \multicolumn{2}{c|}{ St1 } & \multicolumn{2}{c|}{ St2 } \\
\cline { 2 - 5 } \multicolumn{1}{c|}{} & SO & WP & SO & WP \\
\hline FCS & $S_{1}$ & $S_{3}$ & $S_{5}$ & $S_{7}$ \\
\hline CCS & $S_{2}$ & $S_{4}$ & $S_{6}$ & $S_{8}$ \\
\hline
\end{tabular}

Table 1: The eight different corn silage types. They are denoted by $\left(S_{i}\right)_{i=1 \ldots 8}$. Two stages of age (stages St1 and St2), two types of chopping (fine corn silage FCS or coarse CCS), and two types of mixes (only stalk SO and the whole plant WP)

Multi-scale Analysis. The imsfBm model incites us to extend the estimators curves for wide scales. However, in the case of natural texture images, these curves present, in general, more than two regimes. In this case, the computed value of $H$ or $p$, obtained by linear regression, has no clear significance. The estimation of the parameters of the imsfBm then consists in considering all the curve for wide scales (for example 1 to 150 in the case of $512 \times 512$ images). The regression is no longer computed. The estimation result is the curve.

Using this technique allows judicious choice of the scales of study. Two textures can be different only at certain scales. The approach we describe here hinges on this choice of significant scales.

\section{Application: Classification of Corn Silage}

\subsection{Introduction}

The aim of this section is to classify corn silage images. We chose eight silage types, set out in Table 1, having several characteristics. The silages are designated $\left(S_{i}\right)_{i=1 \ldots 8}$ : two stages of age (stages St1 and St2), two types of chopping (fine corn silage FCS or coarse CCS), and two types of mixes (only stalk SO and the whole plant WP).

30 images of each type of silage were recorded (in the same acquisition conditions) for the learning process, plus 10 more images for the classification process. The images used for the classification were not used in the learning process.

\subsection{Learning and Classification Procedures}

To classify the images, it is first necessary to learn how to characterize each class. In the old approach (based on the $\mathrm{fBm}$ model), the class is characterized by the mean values of $H$ and $p$ (computed by a linear regression on the estimation curves between arbitrary chosen scales 1 and 5). These values are the centers of gravity of the class. In our new approach, these centers of gravity are the mean estimation curves (see Figure 1) of the class.

The eight silages types are firstly "learned", using 30 images of each silage class for the learning process.

Then, the six different classes of silages (Stages 1 and 2, with or without corn grain and fine or coarse corn silage) are also "learned". Each class of this segmentation contains 4 silage types (for example silages $S_{1}, S_{3}, S_{5}$ and $S_{7}$ all belong to the class FCS), i.e., 120 


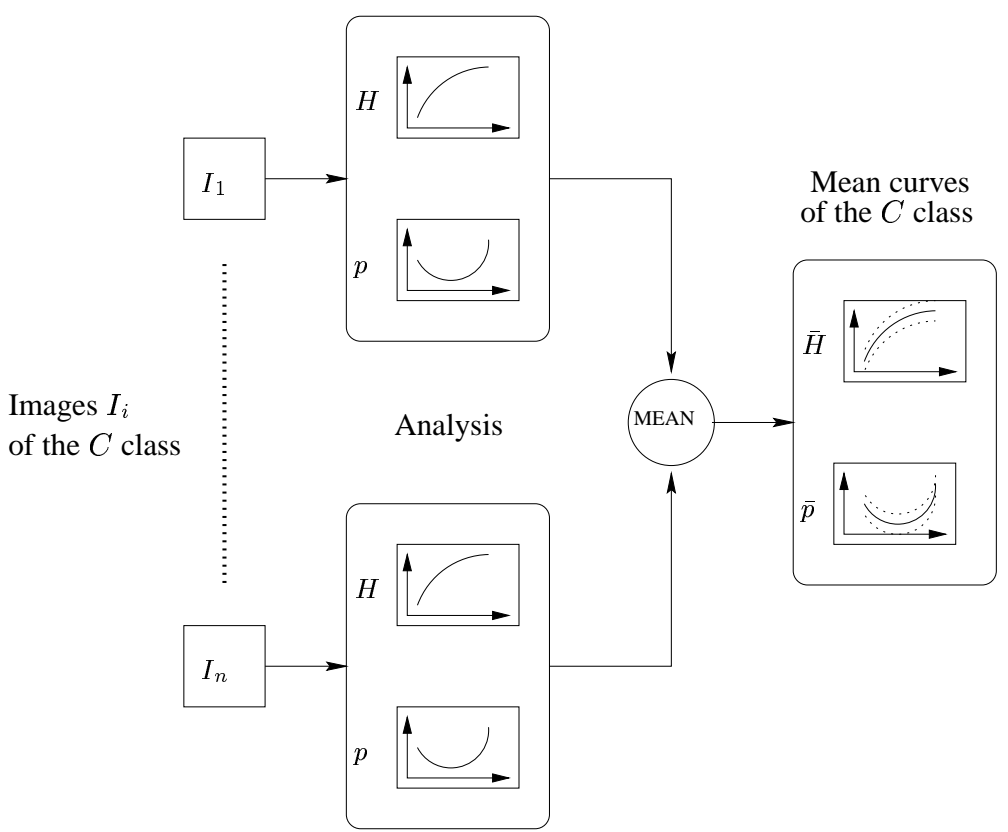

Figure 1: The learning process in our approach: each image $I_{i}$ of a class $C$ is analyzed. The resulting multi-scale estimation curves (for $H$ and $p$ ) are used to produce the mean curves of the class. These curves are the centers of gravity of the class.

images for the learning process. In doing this, we focus on a single criterion by averaging the others.

Figure 2 shows some of the $H$ and $p$-curves of the silages. The $\Delta H$-curve and $\Delta p$ curve are also displayed, these curves being the "derivative" curves of the mean $H$ and $p$-curves $\left(\left(\Delta x_{i}\right)_{i=1 \ldots n-1}=\left(x_{i+1}-x_{i}\right)_{i=1 \ldots n-1}\right)$, and giving information on the regimes (slopes) of the curves.

Simple Classification. The center of gravity of a class is in this case the average computed value of $H$ or $p$ by a linear regression on the estimation curves between scales 1 to 5 . Let $I$ be the image to be classified in one class among $n$ classes $\left(C_{i}\right)_{i=1 \ldots n}$. After the choice of a feature $f$ ( $H$ or $p$ ), the classification procedure returns the class $C_{j}$ such that:

$$
\left|f(I)-f\left(C_{j}\right)\right|=\min _{i=1 \ldots n}\left\{\left|f(I)-f\left(C_{i}\right)\right|\right\} .
$$

Inter-curve Distance based Classification. The center of gravity of a class is in this case the average estimation curves of $H$ or $p$. Let $I$ be the image to be classified in one class among $n$ classes $\left(C_{i}\right)_{i=1 \ldots n}$. After the choice of a distance $d$ and a feature $f$ ( $H$ or $p$ ), depending on a scale interval $D_{S}=\left[S_{\min }, S_{\max }\right]$, the classification procedure returns the class $C_{j}$ such that:

$$
d\left[f_{D_{S}}(I), f_{D_{S}}\left(C_{j}\right)\right]=\min _{i=1 \ldots n}\left\{d\left[f_{D_{S}}(I), f_{D_{S}}\left(C_{i}\right)\right]\right\} .
$$




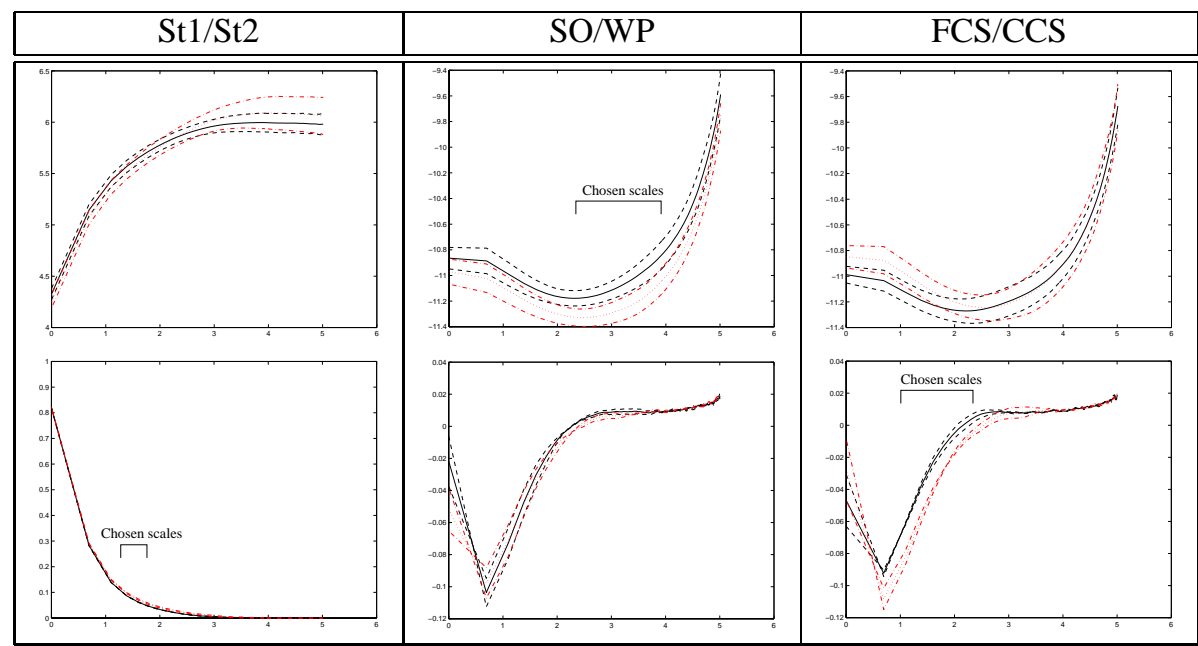

Figure 2: The mean $H$ and $p$-curves ( \pm the standard deviation) of the silages to be compared, and the mean $\Delta H$ and $\Delta p$-curves. These curves are the "derivative" curves of the mean $H$ and $p$-curves $\left(\left(\Delta x_{i}\right)_{i=1 \ldots n-1}=\left(x_{i+1}-x_{i}\right)_{i=1 \ldots n-1}\right)$. Continuous and dashed: St1, SO and FCS. Dotted and dot-dashed: St2, WP and CCS.

Let $\left(x_{i}\right)_{i=1 \ldots n}$ and $\left(y_{i}\right)_{i=1 \ldots n}$ be two curves of $n$ points. We define the following inter-curve distances:

$$
\begin{gathered}
d_{1}(x, y)=\left[\frac{1}{n} \sum_{i=1}^{n}\left|x_{i}-y_{i}\right|^{2}\right]^{\frac{1}{2}} \quad x \in \mathbb{R}^{d}, \\
d_{2}(x, y)=\left[\frac{1}{n-1} \sum_{i=1}^{n}\left|\left(x_{i+1}-x_{i}\right)-\left(y_{i+1}-y_{i}\right)\right|^{2}\right]^{\frac{1}{2}} \quad x \in \mathbb{R}^{d}, \\
d_{3}(x, y)=\left[\frac{1}{n-1} \sum_{i=1}^{n}\left|\left(x_{i+1}-x_{i}\right)-\left(y_{i+1}-y_{i}\right)\right|^{2}+\left|x_{i}-y_{i}\right|^{2}\right]^{\frac{1}{2}} \quad x \in \mathbb{R}^{d},
\end{gathered}
$$

The distance $d_{1}(x, y)$ is a simple Euclidean point-to-point distance, but $d_{2}(x, y)$ is not a distance. It is the distance $d_{1}$ for the curves $\Delta x$ and $\Delta y: d_{2}$ takes into account the slopes of the curves.

The distance $d_{3}(x, y)$ is a "combined" version of $d_{1}$ and $d_{2}$.

\subsection{Results}

This section reports the results obtained using the old and new methods, trying to discriminate between each characteristic two by two.

Using the old method (based on a single index as a signature) and after an empirical choice of scales 1 to 5 for the computation of the linear regression giving the estimated 


\begin{tabular}{|c|c|c|c|c|c|c||c|}
\hline \multicolumn{2}{|c|}{ Scales } & Distance & \multicolumn{2}{|c|}{ Rec. } & Diff. & Total & $\begin{array}{c}\text { Total } \\
\text { Min }\end{array}$ \\
\cline { 4 - 5 } & Max & used & St1 & St2 & & $/ 80$ & percentage \\
\hline \hline 4 & 6 & $d_{3}$ & 33 & 36 & 3 & 69 & 86.25 \\
4 & 6 & $d_{2}$ & 38 & 17 & 21 & 55 & 68.75 \\
4 & 6 & $d_{1}$ & 21 & 30 & 9 & 51 & 63.75 \\
2 & 50 & $d_{3}$ & 26 & 21 & 5 & 47 & 58.75 \\
1 & 7 & $d_{3}$ & 38 & 6 & 32 & 44 & 55 \\
\hline
\end{tabular}

Table 2: Results of classification St1/St2 with the $\Delta H$-curve. Are indicated the number of recognized ("Rec.") silages, the difference $\left|\operatorname{Rec}_{S t 1}-\operatorname{Rec}_{S t 2}\right|$ to give an idea of the robustness of the recognition, the total, and the total percentage.

values of $H$ and $p$, the results are very poor: all 80 images are classified in only one class. For example, in the case of the $\mathrm{St} 1 / \mathrm{St} 2$ classification, the procedure classifies all the silages in class St1.

The results obtained using the new method are presented below.

St1/St2. After observation of the estimation curves, we chose the $\Delta H$-curve as the center of gravity of the classes between scales 4 to 6 , using a distance collecting the curve slopes (actually, the $p$-curve does not give good results in this case): in this region, the two curves ( \pm the standard deviation) do not intersect.

The results obtained are given in Table 2.

The best results are obtained for scales 4 to 6 , using distance $d_{3}$. These scales are small, and correspond to the high frequency content of the textures. This zone is the only one concerned by maturity: the age difference only involves a color change, and does not modify the global aspect of the texture.

It is interesting to note how a slight change in the estimation scales dramatically changes the result: using the distance $d_{3}$ between scales 1 to 7 , the total percentage falls from 86.25 (for the scales 4 to 6 ) to 55 percent.

SO/WP. After observation of the estimation curves, we chose the $p$-curve as the center of gravity of the classes between scales 10 and 30, using a distance collecting the Euclidean differences of the curves (the $H$-curve does not give good results in this case): in this region, the two curves do not intersect.

The results obtained are given in Table 3 .

Between scales 2 to 50 , using distance $d_{1}$, the results are already highly satisfactory (93.75 percent). If we reduce the study interval between scales 10 to 50, using the same distance $d_{1}$, we obtain the same result. This implies the information we attempt is not located in the high frequency content of the texture: the presence of corn grains (in the case "WP" whole plant) does not influence the texture in the high frequencies, because at this observation length and with our camera settings, the grain size generally oscillates between 10 and 50 pixels (this is confirmed by direct measurements).

Between scales 3 and 10, the recognition percentage falls to 80 percent: the information is not in the high frequencies. This again illustrates the usefulness of a multifrequency analysis. 


\begin{tabular}{|c|c|c|c|c|c|c||c|}
\hline \multicolumn{2}{|c|}{ Scales } & Distance & \multicolumn{2}{|c|}{ Rec. } & Diff. & Total & $\begin{array}{c}\text { Total } \\
\text { percentage }\end{array}$ \\
\cline { 1 - 1 } Min & Max & used & SO & WP & & /80 & perann \\
\hline \hline 10 & 30 & $d_{1}$ & 40 & 36 & 4 & 76 & 95 \\
30 & 50 & $d_{1}$ & 40 & 36 & 4 & 76 & 95 \\
10 & 50 & $d_{1}$ & 40 & 35 & 5 & 75 & 93.75 \\
2 & 50 & $d_{1}$ & 40 & 35 & 5 & 75 & 93.75 \\
3 & 10 & $d_{1}$ & 31 & 33 & 2 & 64 & 80 \\
10 & 30 & $d_{2}$ & 36 & 25 & 11 & 61 & 76.25 \\
\hline
\end{tabular}

Table 3: Results of classification SO/WP with the $p$-curve.

\begin{tabular}{|c|c|c|c|c|c|c||c|}
\hline \multicolumn{2}{|c|}{ Scales } & Distance & \multicolumn{2}{c|}{ Rec. } & Diff. & Total & $\begin{array}{c}\text { Total } \\
\text { Min }\end{array}$ \\
\cline { 1 - 1 } & Max & used & FCS & CCS & & /80 & percentage \\
\hline \hline 3 & 9 & $d_{2}$ & 32 & 36 & 4 & 68 & 85 \\
3 & 8 & $d_{2}$ & 30 & 36 & 6 & 66 & 82.5 \\
4 & 9 & $d_{2}$ & 33 & 32 & 1 & 65 & 81.25 \\
3 & 9 & $d_{1}$ & 32 & 28 & 4 & 60 & 75 \\
\hline
\end{tabular}

Table 4: Results of classification FCS/CCS with the $p$-curve.

If we reduce the intervals between 10 and 30 , and between 30 and 50, we obtain an interesting result: the classification percentage is equivalent and slightly greater. Although this difference is not significant, this implies these zones are not only carrying discriminating information, but are also not disturbed by less discriminating zones. The two intervals $[10,30]$ and $[30,50]$ give better results than the "fusion" interval $[10,50]$ : we have multi-discriminant zones.

We can also remark the fundamental importance of the use of the correct distance: between scales 10 and 30 (for which the results are excellent using distance $d_{1}$ ), using distance $d_{2}$ causes the result to fall from 95 to 76.25 percent.

The classification SO/WP is perhaps the easiest case, but the fractal dimension seems unable to discriminate between the two classes. The intermittency parameter, used as an estimation curve, is sometimes more suited than the fractal dimension to analyzing certain physical phenomena [7].

FCS/CCS. After observation of the estimation curves, we chose the $\Delta p$-curve as the center of gravity of the classes between scales 3 and 9, using a distance collecting the curves slopes (the $H$-curve does not give good results in this case): in this region, the two curves do not intersect.

The results obtained are given in Table 4.

The best result is obtained using the curve between scales 3 and 9 and the distance $d_{2}$, with 85 percent correct classification. Here again, if we slightly change the estimation scales ( 4 to 9 and 3 to 8 ), the results are modified ( 82.5 and 81.25 percent).

The choice of the correct distance is again fundamental: between scales 3 and 9 , using distance $d_{1}$ (this distance does not collect the slopes of the curves), the result falls from 85 to 75 percent. 
Old Method Revisited. Given the above results, and if we compute the values of $H$ and $p$ by linear regression in the correct ranges of scales, the results are greatly improved:

Between scales 4 and 6, the mean observed $H$ values for silage types "St1" and "St2" are respectively 0.366 and 0.416 . The classification between these scales gives satisfactory results: $35+31=66 / 80$ correctly recognized silages, i.e., 82.5 percent.

Between scales 10 and 30, the mean observed $p$ values for the silage type "SO" and "WP" are respectively 1.16 and 1.10 (these values have no physical significance, as explained in section 2). The classification between these scales gives satisfactory results: $31+26=57 / 80$ correctly recognized silages, i.e., 71.25 percent.

Between scales 3 and 9, the mean observed $p$ values for the silage type "FCS" and "CCS" are respectively 0.878 and 0.801 . The classification between these scales gives satisfactory results: $32+31=63 / 80$ correctly recognized silages, i.e., 78.5 percent.

\section{Conclusion and Perspectives}

Textures of the real world are difficult to handle with simple fractal models. An effective and robust characterization process must rely upon flexible and effective models. The intermittent multi-scale fractional Brownian motion model presented here is a first step toward a powerful and flexible texture model.

While classical texture analysis often describes the texture too partially and sometimes inaccurately, the multi-scale estimation of the imsfBm parameters does not limit the result to a single value: the multi-scale analysis presented in this paper provides a curve, and this curve is the multi-scale characterization of the texture. The proposed analysis considers the structural aspect of the texture, which is implicitly prohibited by traditional fractal methods of texture analysis.

The experiments carried out on the silage textures images moreover demonstrate the usefulness of a parameter such as intermittency in fractal analysis: the intermittency is not only identifiable, but it also represents a meaningful texture parameter, giving different information from the fractal dimension.

It will be interesting to compare the results of this preliminary work with other results of more traditional and not necessarily fractal methods. A multi-scale extension of other types of analysis can also be considered. The analysis based on cooccurrence matrices for example, which also depends on an observation scale, might allow a multi-scale extension of some Haralick parameters.

More technically, the method could take into account certain multi-discriminant zones: the discriminating information of a texture can be spread across several ranges of scales. Automatic choice of relevant and discriminating scales could be interesting. Moreover, the use of data fusion techniques would provide a useful quality factor, while allowing the processing of several regions of interest and several characteristics.

\section{References}

[1] A. Benassi, S. Cohen, S. Deguy, and J. Istas. Self-similarity and intermittency. In NSF - CBMS - Research conference on wavelets. University of Central Florida. (in press). Available on http://llaic3.uclermont1.fr/ deguy/publi/ss_interm/ss_interm.ps.gz. 
[2] A. Benassi, S. Cohen, J. Istas, and S. Jaffard. Identification of filtered white noises. Stochastic Processes and Applications, (75):31-49, 1998.

[3] A. Benassi and S. Deguy. Multi-scale fractional Brownian motion: definition and identification. Technical Report 83, LLAIC (Laboratoire de Logique, Algorithmique et Informatique de Clermont 1), France, Septembre 1999. Available on http://llaic3.u-clermont1.fr/prepubli/prellaic83.ps.gz.

[4] T. Château, A. Benassi, and P. Bonton. Fractal textures and quality : application to soil superficial structure estimation. In International Conference on Quality Control by Artificial Vision, QCAV97, pages 319-323, Le Creusot, 1997.

[5] J-F. Coeurjolly. Etude empirique de l'estimation du paramètre de Hurst régissant le mouvement Brownien fractionnaire. Technical report, IMAG/LMC, Université Joseph Fourier, Grenoble, 1998.

[6] J.L. Collins and C.J. Deluca. Open loop and closed loop control of posture: a random walk analysis of center of pressure trajectories. Exp. Brain Res., pages 308-318, 1993.

[7] S. Deguy. L'intermittence comme nouveau paramètre de texture. In C. Debain and P. Bonton, editors, Journée Texture en Imagerie Artificielle. CEMAGREF, 5 Octobre 1999. (to appear). Available on http://llaic3.uclermont1.fr/ deguy/publi/jourtex/jourtexture.ps.gz.

[8] M. Dekking, J. Lévy-Véhel, E. Lutton, and C. Tricot, editors. Fractals: theory and applications in engineering. Springer, 1999.

[9] K. Kpalma. Analyse fractale de textures naturelles dans un contexte multirésolution : application à la segmentation d'images multirésolution. $\mathrm{PhD}$ thesis, Institut National des Sciences Appliquées de Rennes, Février 1992.

[10] J. Lévy-Véhel. Fractal approaches in signal processing. Fractals, 3(4):755-775, 1989.

[11] T. Lundhal, W. J. Ohley, S. M. Kay, and R. Siffert. Fractional Brownian motion: a maximum likehood estimator and its application to image texture. IEEE Transactions on Medical Imaging, MI5(3):152-161, September 1986.

[12] B. Mandelbrot and J. Van Ness. Fractional Brownian motion, fractional noises and applications. SIAM Reviews, (10):422-437, 1968.

[13] L. Pothuaud, E. Lespessailles, R. Harba, R. Jennane, V. Royant, E. Eynard, and C.L. Benhamou. Fractal analysis of tabrecular bone texture on radiographs : Discriminant value in postmenopausal osteoporosis. Osteoporosis International, (8):618-625, 1998.

[14] M. Turner, J. Blackledge, and P. Andrews. Fractal geometry in digital imaging. Academic Press Professional, 1998. 